\title{
New fuzzy EWMA control charts for monitoring phase II fuzzy profiles
}

\author{
Ghazale Moghadam $^{a^{*}}$, Gholam Ali Raissi Ardalia and Vahid Amirzadeh ${ }^{\mathrm{b}}$
}

${ }^{a}$ Department of Industrial Engineering Isfahan University Of Technology, Isfahan, Iran

${ }^{b}$ Faculty of mathematics and computer, Shahid Bahonar University Of Kerman, Kerman, Iran

\section{H R O N I C L E}

\begin{tabular}{l} 
Article history: \\
Received June 25, 2015 \\
Received in revised format: \\
June 28, 2015 \\
Accepted July 27, 2015 \\
Available online \\
July 292015 \\
\hline Keywords: \\
Fuzzy set \\
Profile monitoring \\
Fuzzy EWMA control charts
\end{tabular}

\begin{abstract}
A B S T R A C T
In many quality control applications, the quality of a process or product is explained by the relationship between response variable and one or more explanatory variables, called a profile. In this paper, a new fuzzy EWMA control chart for phase II fuzzy profile monitoring is proposed. To this end, we extend EWMA control charts to its equivalent Fuzzy type and then implement fuzzy ranking methods to determine whether the process fuzzy profile is under or out of control. The proposed method is capable of identifying small changes in process under condition of process profile explaining parameters vagueness, roughness and uncertainty. Determining the source of changes, this method provides us with the possibility of recognizing the causes of process transition from stable mode, removing these causes and restoring the process stable mode.
\end{abstract}

\section{Introduction}

Quality of manufacturing products and services is the primary key factor for the success and competitiveness of organizations and there are literally different definitions of quality. Based on the definition of Montgomery (2009), quality is characterized as "inversely proportional to variability". This definition of quality is rooted in the belief that an increase in the variability of key characteristics of a product or service results in a reduction in its quality. Hence, quality control techniques, especially Statistical Process Control (SPC), have absorbed significant amount of attention as an effective tool in reducing variability of processes and improving quality. Although it is usually assumed that the quality of a process can be characterized by one or more quality characteristics and controlled by means of univariate or multivariate control charts, in many applications, the quality of a product or service is better characterized by a functional relationship between a dependent variable and one or more independent ones. The relationship between independent and dependent variables is known as a "Profile". Monitoring profiles, like other process control methods, consists of two phases. The main

\footnotetext{
* Corresponding author.

E-mail address: g.moghadam@in.iut.ac.ir (G. Moghadam) 
goal of the first phase is to evaluate the stability of the process using historical data, and having excluded out-of-control data, to estimate unknown parameters of the profile. In phase II, however, the goal is to promptly identify changes in the process's profile and to discern the underlying reasons responsible for the profile to get out of control. Due to the presence of vagueness, roughness, and uncertainty in real-world applications, the need for methods with which such vagueness can be modeled is obvious. Fuzzy sets theory is a powerful tool in handling real-world uncertainty. There has been a wide range of research regarding phase I and phase II crisp profile monitoring. In this section, researches in phase II linear profiles are reviewed. Proposed solutions in phase II linear profile monitoring can be divided into two categories: Omnibus Control Charts and Individual Control Charts (Noorossana et al., 2011).

The first group of methods monitors the simultaneous changes in profile parameters. Croarkin and Varner (1982) proposed a method based on the principle of inverse calibration, which is now a part of "Linear Calibration using Reference Material” standard (ISO 5725-6 1994). Kang and Albin (2000) suggested two types of control charts for phase II monitoring, one based on $\mathrm{T}^{2}$ statistic and the other based on Exponential Weighted Moving Average (EWMA).

Noorossana et al. (2004) suggested the simultaneous use of MCUSUM, for monitoring the combination of slope and intercept of profile, and R, for monitoring standard deviation. Zou et al. (2006) used Change Point method assuming that model parameters are unknown, but historical in-control data is available for parameter estimation. They applied Likelihood Ratio statistic to investigate the changes in model parameters. Niaki et al. (2007) suggested the use of Generalized Linear Test (GLT) along with R control charts. Zou et al. (2007) applied self-starting control chart approach to monitor phase II profiles. Zhang et al. (2009) presented Likelihood Ratio-based control charts by integrating the EWMA procedure to determine shifts in the intercept, slope, or standard deviation. Li et al. (2010) proposed Variable Sampling Interval (VSI) EWMA method. Noorossana et al. (2010) extended the Kang and Albin's method (2000) to apply under multivariate simple linear profile. Eyvazian et al. (2011) proposed four methods based on likelihood ratio method to monitor multivariate multiple linear profiles in Phase II. Hosseinifard et al. (2011) suggested the use of neural networks for linear profile monitoring. They proposed three methods, including ANN1 and ANN-a in Omnibus control charts, and 3ANN for the second type of methods.

Methods of the second category use individual control charts to monitor profile parameters. All these methods are based on the methods proposed by Kim et al. (2003). They showed that by coding values of independent variables, it is possible to determine independent estimators for profile parameters. Then, they suggested individual EWMA control charts for each parameter. Saghaie et al. (2009) suggested that in Kim et al. (2003) model, instead of EWMA, CUSUM statistic can be used. The use of fuzzy inference systems and adaptive neural networks in monitoring phase II profiles was proposed by Fazel Zarandi and Alaeddini (2010).

In all above mentioned researches on phase II profile monitoring, the primary assumption is that the process's profile is known or can be estimated by means of in-control data from phase I. In both categories, profile parameters are assumed to be crisp and their real nature, which is vague and uncertain, is disregarded. The vagueness of parameters, however, makes the assumption of crisp parameters unrealistic. Uncertainty, roughness, and vagueness of data, which are the bases of analysis of profiles in phase I and phase II, should be included in modeling and analysis. Fuzzy sets theory is an effective tool in bringing vagueness and uncertainty into analyses. Few studies have already been published in the field of fuzzy linear profiles, two studies in phase I and one study in phase II. Noghondarian and Ghobadi (2012) proposed a univariate approach for monitoring phase I fuzzy quality profiles. Ghobadi et al. (2012) proposed a multivariate approach for monitoring process/product fuzzy quality profiles in phase I. Moghadam et al. (2015) developed two methods based on fuzzy $T^{2}$ Hotelling and fuzzy EWMA control charts for phase II fuzzy linear profiles. They assumed that the values of 
dependent variable are fuzzy. In this paper, a new method of phase II profile monitoring is proposed. It is assumed in the proposed model that the functional relationship between independent and dependent variables of the process profile is vague and defined by linear fuzzy regression. Fuzzy EWMA statistics are used in order to monitor each one of the profile parameters.

The organization of the paper is as follows: in section 2, basic concepts of fuzzy set theory are briefly reviewed. In section 3, fuzzy linear profiles, their assumptions, and the fuzzy regression model for identifying fuzzy profiles of samples are described. In section 4, fuzzy EWMA statistics and their calculations are defined and the proposed method for drawing and analyzing control charts based on these statistics are discussed. Section 5, demonstrates an example in which the proposed method is employed and its efficiency is studied. In the end, conclusions and possible future researches are provided.

\section{Preliminary concepts}

Definition 2.1. A fuzzy number $\tilde{A}=(a, \lambda, \beta)$ is called an asymmetrical triangular fuzzy number with center $a$, left spread $\lambda$ and right spread $\beta$ if its membership function can be shown as follows:

$$
\mu_{\tilde{A}(x)}=\left\{\begin{array}{cc}
\frac{x-(a-\lambda)}{\lambda} & a-\lambda<x \leq a \\
\frac{(a+\beta)-x}{\beta} & a \leq x \leq a+\beta \\
0 & \text { OtherWise }
\end{array}\right.
$$

If right and left spreads of the triangular fuzzy number are given as $(\lambda=\beta=S)$, the triangular fuzzy number is called symmetric and shown as $(a, s)$ where $a$ is the number's center and $s$ is its spread. A typical triangular fuzzy number is shown in Fig. 1.

Definition 2.2. $\alpha$-Cut of fuzzy set $\widetilde{\mathrm{A}}$ is a crisp set including elements from reference set $\mathrm{X}$ with membership degree in fuzzy set $\widetilde{\mathrm{A}}$ of at least as big as $\alpha(0<\alpha \leq 1)$. We denoted the $\alpha$-Cut of fuzzy set $\tilde{A}$ by $\operatorname{Cut}_{\alpha}(\tilde{A})$,

$$
\operatorname{Cut}_{\alpha}(\tilde{A})=\left\{x \in X \mid \mu_{\tilde{A}(x)} \geq \alpha\right\}
$$

If $\widetilde{\mathrm{A}}$ is an asymmetrical triangular fuzzy number, $\mathrm{Cut}_{\alpha}(\tilde{A})$ is:

$$
\operatorname{Cut}_{\alpha}(\tilde{A})=[(\alpha-1) \lambda+a,(1-\alpha) \beta+a]=\left[a^{L}(\alpha), a^{R}(\alpha)\right]
$$

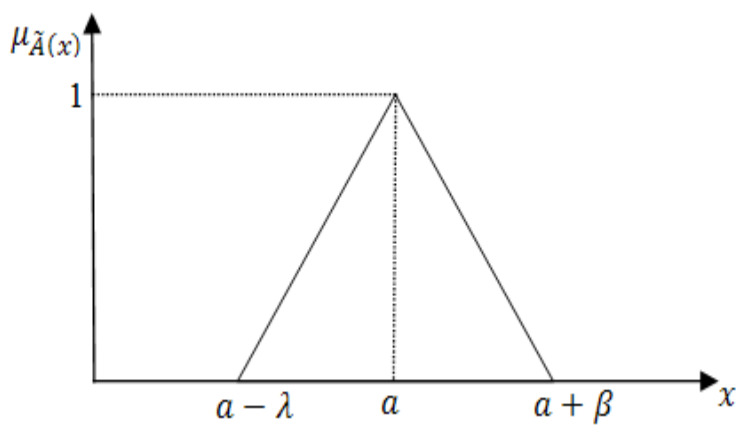

Fig. 1. Triangular fuzzy number 


\section{Fuzzy linear profiles}

In phase II study of linear fuzzy profiles, online data, acquired from the examination of the process, are analyzed. The purpose of this phase is to promptly determine if the parameters of the profile are out of control. It is assumed in this phase that profile parameters are known or can be estimated by means of in-control historical data set. Due to the fact that in real-world cases, information is incomplete, vague or approximate, we introduce fuzzy linear profile monitoring in phase II and assume that the parameters of the process's profile are triangular fuzzy numbers. In this section, first the assumptions of fuzzy linear profile are described, and then the fuzzy regression model for identifying fuzzy linear profile of samples is explained.

\subsection{Assumptions}

Assume that the $j$ th random sample acquired over time is demonstrated by $\left(x_{i}^{\prime}, y_{i j}\right) i=1,2, \ldots, n j=$ $1,2, \ldots, m$. If the process is in-control, the fuzzy linear profile that relates dependent variable $Y$ to independent variable $X^{\prime}$ is:

$$
\tilde{Y}_{i j}=\tilde{B}_{0}+\tilde{B}_{1} X_{i}^{\prime} \quad i=1,2, \ldots, n, j=1,2, \ldots, m
$$

In Eq. (4), it is assumed that independent variable $X_{i}^{\prime}$ has non-fuzzy and fixed values from one sample to another. In phase II profile monitoring, it is assumed that in-control values of the parameters, $\widetilde{B}_{0}, \widetilde{B}_{1}$ are either known numbers or can be estimated by means of in control historical data. $\tilde{B}_{0}$ and $\tilde{B}_{1}$ are triangular fuzzy numbers. In our proposed method, values of independent variable $\left(X_{i}^{\prime}\right)$ are coded in a way that the average of coded variables is zero. This will make the analysis easier, as the coding turns parameter estimators of profiles into mutually independent estimators (Kim et al., 2003. Coding is performed by Eq. (5). In Eq. (5), $\bar{X}$ is the average of independent variable's values.

$$
X_{i}=X_{i}^{\prime}-\bar{X}
$$

As a result, the linear regression model will be:

$$
\begin{aligned}
& \widetilde{\mathrm{Y}}_{\mathrm{ij}}=\widetilde{\mathrm{A}}_{0}+\tilde{A}_{1} X_{i} \quad i=1,2, \ldots, n, j=1,2, \ldots, m \\
& \text { where } \tilde{A}_{0}=\widetilde{B}_{0}+\tilde{B}_{1} \bar{X}, \tilde{A}_{1}=\widetilde{B}_{1} .
\end{aligned}
$$

\subsection{Determining profile of samples using fuzzy linear regression}

The first step in our phase II profile monitoring method, is to calculate profile parameters of $m$ random samples. Afterwards, a procedure should be developed in order to examine the state of the profile (whether it is in control or out of control). In this paper, fuzzy regression model in (Bucklly, 2006) is used in order to calculate the profile of each random sample. In this method, $\alpha$-cut and interval algebra are employed to specify fuzzy membership functions of the parameters of linear regression. The $\alpha$-cut of the fuzzy membership function of each parameter of linear regression is assumed to be conformant with $(1-\alpha) 100 \%$ confidence interval of that parameter. Base on this method, the estimated value of the fuzzy parameters of model (6), are defined by Eq. (7) to Eq. (13). (All the parameters which are

shown by $\left({ }^{\top}\right)$ symbol are deterministic estimators, while parameters depicted by $(\widetilde{\sim})$ symbol are fuzzy estimators.)

\subsubsection{Error variance membership function at $\alpha$-level:}

$\operatorname{Cut}_{\alpha}\left(\tilde{\sigma}_{j}^{2}\right)=\left[\sigma_{j L}^{2}(\alpha), \sigma_{j R}^{2}(\alpha)\right]=\left[\frac{n \widehat{\sigma}_{j}^{2}}{L(\lambda)}, \frac{n \widehat{\sigma}_{j}^{2}}{R(\lambda)}\right]$,

where $L(\lambda)=(1-\lambda) \chi_{R, 0.005}^{2}+\lambda n$ and $R(\lambda)=(1-\lambda) \chi_{L, 0.005}^{2}+\lambda n . \quad 0 \leq \lambda \leq 1$. 
and $l=\int_{0}^{R(\lambda)} \chi^{2} d x$ and $r=\int_{L(\lambda)}^{\infty} \chi^{2} d x$ and $\alpha=l+r$.

and $\hat{\sigma}_{j}^{2}=\left(\frac{1}{n}\right) \sum_{i=1}^{n}\left(y_{i j}-\hat{a}_{\mathrm{j}}-\hat{b}_{\mathrm{j}}\left(x_{i j}-\bar{x}_{j}\right)\right)^{2}$.

In the above mentioned equations, $\chi_{R, 0.005}^{2}$ and $\chi_{L, 0.005}^{2}$ are, respectively, points on the right side and the the left side of the $\chi^{2}$ distribution with $(n-2)$ degrees of freedom, which satisfy the following equation:

$$
p\left(X \geq \chi_{R, \frac{\alpha}{2}}^{2}\right)=p\left(X \leq \chi_{L, \frac{\alpha}{2}}^{2}\right)=\frac{\alpha}{2}
$$

\subsubsection{Membership function of intercept at $\alpha$-level:}

$$
\operatorname{Cut}_{\alpha}\left(\tilde{a}_{0 j}\right)=\left[a_{0 j L}(\alpha), a_{0 j R}(\alpha)\right]=\left[\hat{a}_{0 j}-t_{\frac{\alpha}{2}} \sqrt{\frac{\widehat{\sigma}_{j}^{2}}{(n-2)}}, \hat{a}_{0 j}+t_{\frac{\alpha}{2}} \sqrt{\frac{\widehat{\sigma}_{j}^{2}}{(n-2)}}\right]
$$

where $\hat{a}_{0 j}=\bar{y}_{j}$.

In the above mentioned equations, $t_{\frac{\alpha}{2}}$ is a point in the t-Student distribution with $(n-2)$ degrees of freedom, which satisfies the following equation,

$$
p\left(X \leq t_{\frac{\alpha}{2}}\right)=\frac{\alpha}{2} \text {. }
$$

3.2.3. Membership function of intercept variance at $\alpha$-level:

$$
\operatorname{Cut}_{\alpha}\left(\tilde{\sigma}_{a_{0 j}}^{2}\right)=\left[\sigma_{a_{0 j} L}^{2}(\alpha), \sigma_{a_{0 j} R}^{2}(\alpha)\right]=\left[n \hat{\sigma}_{j}^{2} /(n-2) L(\lambda), n \hat{\sigma}_{j}^{2} /(n-2) R(\lambda)\right] .
$$

3.2.4. Membership function of slop at $\alpha$-level:

$$
\operatorname{Cut}_{\alpha}\left(\tilde{a}_{1 j}\right)=\left[a_{1 j L}(\alpha), a_{1 j R}(\alpha)\right]=\left[\hat{a}_{1 j}-t_{\frac{\alpha}{2}} \sqrt{\frac{C_{1}}{C_{2}}}, \hat{a}_{1 j}+t_{\frac{\alpha}{2}} \sqrt{\frac{C_{1}}{C_{2}}}\right] \text {, }
$$

where $C_{1}=n \hat{\sigma}^{2} \quad, C_{2}=(n-2) \sum_{i=1}^{n}\left(x_{i}-\bar{x}\right)$ and $\hat{a}_{1 j}=$

$\sum_{i=1}^{n} y_{i j}\left(x_{i j}-\bar{x}_{j}\right) / \sum_{i=1}^{n}\left(x_{i j}-\bar{x}_{j}\right)^{2}$.

\subsubsection{Membership function of slop variance at $\alpha$-level:}

$$
\operatorname{Cut}_{\alpha}\left(\tilde{\sigma}_{a_{1 j}}^{2}\right)=\left[\sigma_{a_{1 j} L}^{2}(\alpha), \sigma_{a_{1 j} R}^{2}(\alpha)\right]=\left[n \hat{\sigma}_{j}^{2} / C_{2} L(\lambda), n \hat{\sigma}_{j}^{2} / C_{2} R(\lambda)\right] .
$$

\section{Monitoring fuzzy linear profiles in phase II}

Having defined a fuzzy profile for every instance of $m$ random samples, we need to decide on performance mode of the process. Is the process profile in correspondence with the under control mode or does ensuring quality of products depend on applying changes and new settings in the process? There are several statistical methods available for monitoring crisp profiles in phase II. Kim et al. (2003) proposed the use of EWMA control charts in order to monitor the parameters of crisp profiles. In this research, we extended this method to fuzzy profiles. Fuzzy Exponentially Weighted Moving Average (FEWMA ) statistic is the fuzzy triangular number that is developed for this purpose. In this section, firstly the method of creating FEWMA control charts are described, and secondly, the method of analysis is discussed. 


\subsection{FEWM fuzzy control charts}

After coding X values, every profile has 3 independent fuzzy parameters. Therefore, each of the profile parameters can be monitored on a separate control chart based on fuzzy EWMA statistic.

\subsubsection{FEWM $A_{I}$ statistic for monitoring intercept of fuzzy linear profile}

Assuming that $\widetilde{\mathrm{A}}_{0}, \widetilde{\mathrm{A}}_{1}$ and $\widetilde{\sigma}_{0}$ are the values of intercept, slop and standard deviation of the process profile in the under control mode and $\left(\tilde{a}_{0 j}, \tilde{a}_{1 j}, \tilde{\sigma}_{j}\right) j=1,2, \ldots, m$ are the equivalent estimator of them respectively. $F \widetilde{E W M} A_{I}$ statistics is defined to monitor the intercept of regression line as follows:

$$
\widetilde{F W M} A_{I(j)}=\theta \tilde{Z}_{j}+(1-\theta) \widetilde{F W M} A_{I(j-1)}
$$

In the above equation, $0<\theta \leq 1$ is a smoothing constant, $\tilde{a}_{0 j}$ is the estimated value of intercept from the jth profile and $\overrightarrow{\mathrm{FEWM}_{\mathrm{I}(0)}}=\tilde{0}$. The parameter $\theta$ is selected so that the control chart produces a particular ARL in control mode. In order to calculate and plot FEWMA statistics, $\alpha$-cut and interval algebra is used. If $\tilde{I}_{j}$ and $\widetilde{Z}_{a j}$ are defined by Eq. (15) to Eq. (18), $\alpha$-cut of $F \widetilde{E W M} A_{I}$ statistic is defined by Eq. (19).

$$
\begin{aligned}
& \tilde{I}_{j}=\tilde{a}_{0 j}-\tilde{A}_{0} \text { and } \operatorname{Cut}_{\alpha}\left(\tilde{I}_{j}\right)=\left[I_{L j}(\alpha), I_{R j}(\alpha)\right] \\
& \text { where } I_{\mathrm{Lj}}(\alpha)=a_{0 j L}(\alpha)-a_{0 R}(\alpha) \text { and } I_{\mathrm{Rj}}(\alpha)=a_{0 j R}(\alpha)-a_{0 L}(\alpha) . \\
& \text { and } \operatorname{Cut}_{\alpha}\left(\tilde{a}_{0 j}\right)=\left[a_{0 j L}(\alpha), a_{0 j R}(\alpha)\right] \text { and } C u t_{\alpha}\left(\tilde{a}_{0}\right)=\left[a_{0 L}(\alpha), a_{0 R}(\alpha)\right] . \\
& \text { If }\left(I_{L j}(\alpha)>0, I_{R j}(\alpha)>0\right) \text { then } C u t_{\alpha}\left(\widetilde{Z}_{a j}\right)=\left[Z_{a j L}(\alpha), Z_{a j R}(\alpha)\right]=\left[\frac{I_{L j}(\alpha)}{\sqrt{\sigma_{a_{j} R}^{2}(\alpha)}}, \frac{I_{R j}(\alpha)}{\sqrt{\sigma_{a_{j} L}^{2}(\alpha)}}\right] \\
& \text { If }\left(I_{L j}(\alpha)<0, I_{R j}(\alpha)>0\right) \text { then } C u t_{\alpha}\left(\tilde{Z}_{a j}\right)=\left[Z_{a j L}(\alpha), Z_{a j R}(\alpha)\right]=\left[\frac{I_{L j}(\alpha)}{\sqrt{\sigma_{a_{j} L}^{2}(\alpha)}}, \frac{I_{R j}(\alpha)}{\sqrt{\sigma_{a_{j} L}^{2}(\alpha)}}\right] \\
& \text { If }\left(I_{L j}(\alpha)<0, I_{R j}(\alpha)<0\right) \text { then } C u t_{\alpha}\left(\tilde{Z}_{a j}\right)=\left[Z_{a j L}(\alpha), Z_{a j R}(\alpha)\right]=\left[\frac{I_{L j}(\alpha)}{\sqrt{\sigma_{a_{j} L}^{2}(\alpha)}}, \frac{I_{R j}(\alpha)}{\sqrt{\sigma_{a_{j} R}^{2}(\alpha)}}\right] \\
& C u t_{\alpha}\left(F E W M A_{\mathrm{Ij})}\right)=\left[F E W M A_{\mathrm{IL}(\mathrm{j})}(\alpha), F E W M A_{\mathrm{IR}(\mathrm{j})}(\alpha)\right] \\
& \text { where } F E W M A_{\mathrm{IL}(\mathrm{j})}(\alpha)=\theta Z_{a j L}(\alpha)+(1-\theta) F E W M A_{\mathrm{IL}(\mathrm{j}-1)}(\alpha) \\
& \text { and } F E W M A_{I R(j)}(\alpha)=\theta Z_{a j R}(\alpha)+(1-\theta) F E W M A_{I R(j-1)}(\alpha) .
\end{aligned}
$$

The control chart would be out of control if $\widetilde{F W M} A_{\mathrm{I}(\mathrm{j})}>\tilde{h}_{I}$ or $\widetilde{F W M} A_{\mathrm{I}(\mathrm{j})}<-\tilde{h}_{I}$ holds. $\tilde{h}_{I}$ (the control limit of the chart) is a fuzzy triangular number and is defined as follows:

$$
\tilde{h}_{I}=\tilde{\sigma}_{0}^{2} L_{I} \sqrt{\theta / n(2-\theta)}
$$

Parameters $\theta$, L should be selected for the result to be in a specific in-control ARL.

\subsection{2. $F \widetilde{E W M} A_{S}$ statistic for monitoring slop of fuzzy linear profile}

$\widetilde{F E W M} A_{S}$ statistic, which is being used to monitor the slope of the regression line, is defined similar to $\widetilde{\text { FWM }} A_{\text {I }}$ by Eq. (21) to Eq. (25). 


$$
\tilde{S}_{j}=\tilde{a}_{1 j}-\tilde{A}_{1}, \operatorname{Cut}_{\alpha}\left(\tilde{S}_{j}\right)=\left[S_{L j}(\alpha), S_{R j}(\alpha)\right],
$$

where $S_{L j}(\alpha)=a_{1 j L}(\alpha)-a_{1 R}(\alpha)$ and $S_{R j}(\alpha)=a_{1 j R}(\alpha)-a_{1 L}(\alpha)$.

and $\operatorname{Cut}_{\alpha}\left(\tilde{a}_{1 j}\right)=\left[a_{1 j L}(\alpha), a_{1 j R}(\alpha)\right]$ and $\operatorname{Cut}_{\alpha}\left(\tilde{a}_{1}\right)=\left[a_{1 L}(\alpha), a_{1 R}(\alpha)\right]$.

If $\left(S_{L j}(\alpha)>0, S_{R j}(\alpha)>0\right)$ then $C u t_{\alpha}\left(\widetilde{Z}_{b j}\right)=\left[Z_{b j L}(\alpha), Z_{b j R}(\alpha)\right]=\left[\frac{s_{L j}(\alpha)}{\sqrt{\sigma_{b_{j} R}^{2}(\alpha)}}, \frac{S_{R j}(\alpha)}{\sqrt{\sigma_{b_{j} L}^{2}(\alpha)}}\right]$

If $\left(S_{L j}(\alpha)<0, S_{R j}(\alpha)>0\right)$ then $C u t_{\alpha}\left(\tilde{Z}_{b j}\right)=\left[Z_{b j L}(\alpha), Z_{b j R}(\alpha)\right]=\left[\frac{S_{L j}(\alpha)}{\sqrt{\sigma_{b_{j} L}^{2}(\alpha)}}, \frac{S_{R j}(\alpha)}{\sqrt{\sigma_{b_{j} L}^{2}(\alpha)}}\right]$

If $\left(S_{L j}(\alpha)<0, S_{R j}(\alpha)<0\right)$ then $C u t_{\alpha}\left(\tilde{Z}_{j}\right)=\left[Z_{b j L}(\alpha), Z_{b j R}(\alpha)\right]=\left[\frac{S_{L j}(\alpha)}{\sqrt{\sigma_{b_{j} L}^{2}(\alpha)}}, \frac{S_{R j}(\alpha)}{\sqrt{\sigma_{b_{j} R}^{2}(\alpha)}}\right]$

$\operatorname{Cut}_{\alpha}\left(\widetilde{F E M} A_{S(j)}\right)=\left[F E W M A_{S L(j)}(\alpha), F E W M A_{S R(j)}(\alpha)\right]$

where $F E W M A_{S L(j)}(\alpha)=\theta Z_{b j L}(\alpha)+(1-\theta) F E W M A_{S L(j-1)}(\alpha)$

and $F E W M A_{S R(j)}(\alpha)=\theta Z_{b j R}(\alpha)+(1-\theta) F E W M A_{S R(j-1)}(\alpha)$

The upper and lower control limit of the control chart based on $\widetilde{F W M} A_{S}$ are $\pm \tilde{h}_{S} \cdot \tilde{h}_{S}$ are fuzzy triangular numbers and they are defined as follows:

$$
\tilde{h}_{S}=\tilde{\sigma}_{0}^{2} L_{S} \sqrt{\theta / n(2-\theta)},
$$

where $\mathrm{L}_{\mathrm{S}}>0$ is chosen to give a specified in-control ARL.

\subsection{3. $\overline{E W M} A_{E(i)}$ statistic for monitoring error variance}

In order to monitor the error variance of the profile, a fuzzy statistic is defined as in Eq. (27):

$$
F \widetilde{E W M} A_{E(j)}=\operatorname{Max}\left\{\theta\left(\operatorname{Ln}\left(\tilde{\sigma}_{j}^{2}\right)\right)+(1-\theta) E \widetilde{E M} A_{E(j-1)}, \operatorname{Ln} \tilde{\sigma}_{0}^{2}\right\}
$$

where $E \widehat{E W M} A_{E(0)}=\operatorname{Ln} \tilde{\sigma}_{0}^{2}$. Upper control limit for monitoring an increase in the variance of the process is defined based on the following equation:

$$
\begin{aligned}
& \widetilde{U C L}=\operatorname{Ln} \tilde{\sigma}_{0}^{2}+l_{E}(\theta /(2-\theta))^{\frac{1}{2}}, \\
& \text { where } l_{E}=\left(\frac{2}{n-2}+\frac{2}{(n-2)^{2}}+\frac{4}{3(n-2)^{3}}-\frac{16}{15(n-2)^{5}}\right) \times L_{E} .
\end{aligned}
$$

In these equations, parameters $L_{E}$ and $\theta$ are chosen in a way that a specific in-control ARL is produced and $\tilde{\sigma}_{0}^{2}$ is the in-control value of $\tilde{\sigma}^{2}$. By means of the method of fuzzy ranking described in the next section, $F \widetilde{E W M} A_{E}$ and upper control limit of the control chart can be calculated.

\subsection{Triangular fuzzy numbers' ranking}

In order to be able to assess the state of the profile (whether it is in control or not) and also for calculating $\mathrm{FEWM}_{\mathrm{E}(\mathrm{j})}$, triangular fuzzy numbers should be compared and ranked. Many methods have been proposed for ranking triangular fuzzy numbers, and in this paper, Bucklly's method (2006) is selected 
because of its simplicity and efficiency. Bucklly (2006) defined $\leq{ }^{1}$ between the fuzzy numbers $\widetilde{M}$ and $\widetilde{\mathrm{N}}$ as follows:

$$
v(\widetilde{M} \leq \widetilde{N})=\max \{\min (\widetilde{M}(x), \widetilde{N}(y)) \mid x \leq y\},
$$

which measures how much $\widetilde{M}$ is less than or equal to $\widetilde{N}$. $\widetilde{N}$ is less than $\widetilde{M}$, if $v(\widetilde{N} \leq \widetilde{M})=1$ but $v(\widetilde{\mathrm{M}} \leq \widetilde{\mathrm{N}})<\eta$, where $\eta$ is some fixed fraction in $[0,1]$ and $\widetilde{\mathrm{M}} \approx \widetilde{\mathrm{N}}(\widetilde{\mathrm{M}}$ is approximately equal to $\widetilde{\mathrm{N}})$ when both $\widetilde{\mathrm{M}}<\widetilde{\mathrm{N}}$ and $\widetilde{\mathrm{M}}>\widetilde{\mathrm{N}}$ are false.

When control chart is drawn, we need to decide on the status of the process profile. The test value $(\tilde{Z})$ should be compared with control limits ( $\widetilde{U C L}, \quad \widetilde{L C L}$ ). Each time the comparison is done, in fact, the statistical hypothesis of $\mathrm{H}_{0}$ : "the profile is in control" is tested against the hypothesis $\mathrm{H}_{1}$ : "the profile is out of control”.

Our final decision (reject, Do not reject) depends on the relationship between $\widetilde{Z}$ and the UCL( $\widetilde{L C L}$ ). In comparing $\tilde{\mathrm{Z}}$ and the $\widetilde{U C L}$, the result is: $\widetilde{\mathrm{Z}}>\widetilde{U C L}$ ( reject $H_{0}$ ), or $\tilde{\mathrm{Z}} \approx \widetilde{U C L}$ ( no decision), or $\tilde{\mathrm{Z}}<\widetilde{U C L}$ ( Do not reject). Similar results are expected when comparing $\widetilde{Z}$ and the $\widetilde{L} \widetilde{L}$. The final decision based on the result of comparisons between $\widetilde{Z}$ and $\widetilde{U C L}(\widetilde{L C L})$, may be one of the following:

1) The final decision is "reject $H_{0}$ ", if $H_{0}$ is rejected based on at least one of the comparisons.

2) The final decision is "Do not reject $H_{0}$ ", if the result of the both comparisons is "Do not reject".

3) The final decision is "no decision", if at least one of the comparison results is "no decision".

The interesting aspect of this fuzzy hypothesis testing is that, the result can be "no decision". This is because of the fuzzy numbers, which incorporate all the uncertainty in the confidence intervals, that make $\widetilde{\mathrm{M}} \approx \widetilde{\mathrm{N}}$ possible for two different fuzzy numbers $\widetilde{\mathrm{M}}$ and $\widetilde{\mathrm{N}}$.

\section{Numerical Example}

For the purpose of illustrating the application of FEWMA charts in phase II profile monitoring, the simulated data set in (Kang \& Albin, 2000) is used (Table 1). This set includes 20 in-control simulated profiles and 9 out of control ones, by increasing the slope of the regression line from 2 to 2.4. Analysis is carried out assuming that the parameters of the in-control fuzzy profile meet Eq. (30) to Eq. (32). Smoothing coefficient is also set to $\theta=0.2$ and $L_{S}=L_{I}=3$. The results of the analysis based on FEWMA control charts are depicted in Figs. (4-6).

$$
\begin{aligned}
& \operatorname{Cut}_{\alpha}\left(\tilde{A}_{0}\right)=[12.95+0.05 \alpha, 13.05-0.05 \alpha] \\
& \operatorname{Cut}_{\alpha}\left(\tilde{A}_{1}\right)=[1.95+0.05 \alpha, 2.05-0.05 \alpha] \\
& \operatorname{Cut}_{\alpha}\left(\tilde{\sigma}_{0}^{2}\right)=[0.7+0.3 \alpha, 1.3-0.3 \alpha]
\end{aligned}
$$

Calculations and drawing graphs of fuzzy profile parameters and also fuzzy statistics of control charts are done by means of MATLAB software. Figs. (2-4) show the plots of triangular fuzzy numbers for intercept, slope, and error variance of profiles 1 to 5 . In these figures, $P_{i} i=1,2, \ldots, 5$ shows profile number $i$. In Figs. (4-6), red triangular fuzzy numbers show the control limits. Based on Fig. 5, it can be inferred that in $\widetilde{F W M} A_{I}$ no out of control signal is observed. In Fig. 6, it is clear that in $\widetilde{F W M} A_{S}$ control chart, there are out of control signals for profiles 27 and up. Fig. 7 shows that there is no out of control signal in $\widetilde{F W M} A_{E}$ plot. The triple control charts show that it is not possible to make decision based on available information from sample number 22 up to 26. 
Table 1

Data Set and results of analysis

\begin{tabular}{|c|c|c|c|c|c|c|c|c|}
\hline \multirow[b]{2}{*}{ Row } & \multirow[b]{2}{*}{-3} & \multirow[b]{2}{*}{-1} & \multirow[b]{2}{*}{1} & \multirow[b]{2}{*}{3} & \multicolumn{4}{|c|}{ Final decision } \\
\hline & & & & & Slop & Intercept & Error variance & Final decision \\
\hline 1 & 6.818 & 12.644 & 12.919 & 19.402 & Do not reject & Do not reject & Do not reject & Do not reject \\
\hline 2 & 7.723 & 11.33 & 16.987 & 20.322 & Do not reject & Do not reject & Do not reject & Do not reject \\
\hline 3 & 5.017 & 10.612 & 13.934 & 20.381 & Do not reject & Do not reject & Do not reject & Do not reject \\
\hline 4 & 7.433 & 10.399 & 16.386 & 17.029 & Do not reject & Do not reject & Do not reject & Do not reject \\
\hline 5 & 6.834 & 11.428 & 16.192 & 19.11 & Do not reject & Do not reject & Do not reject & Do not reject \\
\hline 6 & 7.068 & 12.108 & 14.919 & 19.478 & Do not reject & Do not reject & Do not reject & Do not reject \\
\hline 7 & 8.209 & 11.732 & 14.901 & 19.149 & Do not reject & Do not reject & Do not reject & Do not reject \\
\hline 8 & 8.515 & 10.986 & 15.451 & 18.639 & Do not reject & Do not reject & Do not reject & Do not reject \\
\hline 9 & 8.005 & 9.51 & 15.926 & 18.891 & Do not reject & Do not reject & Do not reject & Do not reject \\
\hline 10 & 6.705 & 11.311 & 14.496 & 17.244 & Do not reject & Do not reject & Do not reject & Do not reject \\
\hline 11 & 6.327 & 9.925 & 14.647 & 20.649 & Do not reject & Do not reject & Do not reject & Do not reject \\
\hline 12 & 6.057 & 9.075 & 14.573 & 18.61 & Do not reject & Do not reject & Do not reject & Do not reject \\
\hline 13 & 9.106 & 10.037 & 17.018 & 18.398 & Do not reject & Do not reject & Do not reject & Do not reject \\
\hline 14 & 7.616 & 11.159 & 15.734 & 18.687 & Do not reject & Do not reject & Do not reject & Do not reject \\
\hline 15 & 6.355 & 10.176 & 13.908 & 19.772 & Do not reject & Do not reject & Do not reject & Do not reject \\
\hline 16 & 6.967 & 12.525 & 14.753 & 17.461 & Do not reject & Do not reject & Do not reject & Do not reject \\
\hline 17 & 9.686 & 11.626 & 15.456 & 17.735 & Do not reject & Do not reject & Do not reject & Do not reject \\
\hline 18 & 5.266 & 12.292 & 16.461 & 18.732 & Do not reject & Do not reject & Do not reject & Do not reject \\
\hline 19 & 7.014 & 9.93 & 15.485 & 18.634 & Do not reject & Do not reject & Do not reject & Do not reject \\
\hline 20 & 5.912 & 10.017 & 13.494 & 17.924 & Do not reject & No decision & Do not reject & No decision \\
\hline 21 & 6.313 & 10.788 & 14.682 & 20.432 & Do not reject & Do not reject & Do not reject & Do not reject \\
\hline 22 & 7.156 & 10.523 & 15.29 & 21.125 & Do not reject & No decision & Do not reject & No decision \\
\hline 23 & 5.498 & 9.941 & 15.313 & 20.957 & No decision & Do not reject & Do not reject & No decision \\
\hline 24 & 5.675 & 10.688 & 15.865 & 18.78 & No decision & Do not reject & Do not reject & No decision \\
\hline 25 & 5.002 & 9.833 & 16.039 & 20.619 & No decision & Do not reject & Do not reject & No decision \\
\hline 26 & 4.009 & 10.267 & 17.464 & 19.556 & No decision & Do not reject & Do not reject & No decision \\
\hline 27 & 4.326 & 10.245 & 16.378 & 21.058 & Reject & Do not reject & Do not reject & Reject \\
\hline 28 & 5.488 & 11.946 & 16.957 & 20.617 & Reject & Do not reject & Do not reject & Reject \\
\hline 29 & 4.361 & 11.675 & 16.583 & 20.282 & Reject & Do not reject & Do not reject & Reject \\
\hline
\end{tabular}

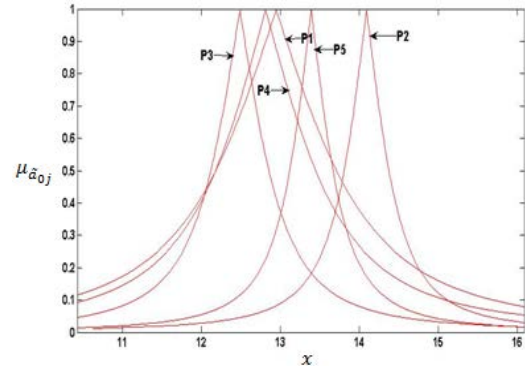

Fig. 2. Triangular fuzzy numbers of the intercepts of profiles 1 to 5

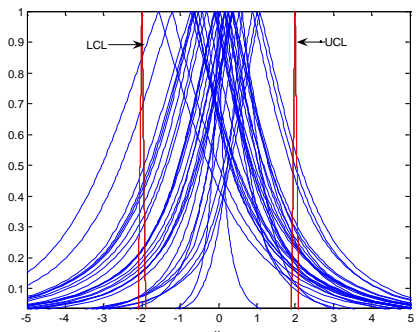

Fig. 5. FEWMA control chart for intercepts

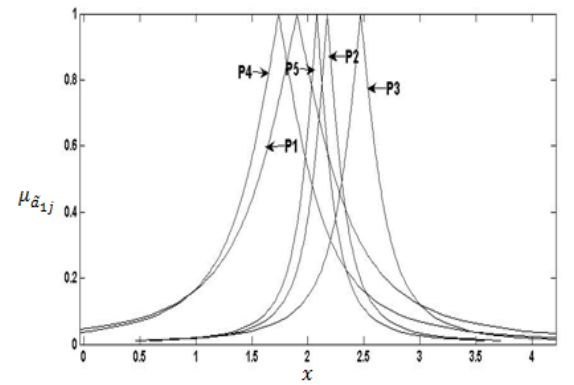

Fig. 3. Triangular fuzzy numbers of the slopes of profiles 1 to 5

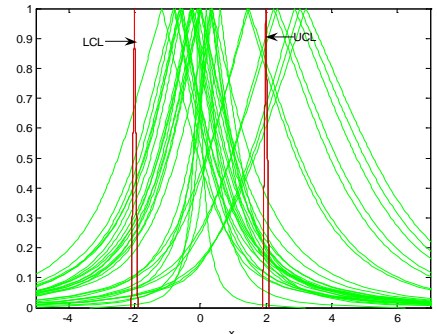

Fig. 6. FEWMA control chart for slopes

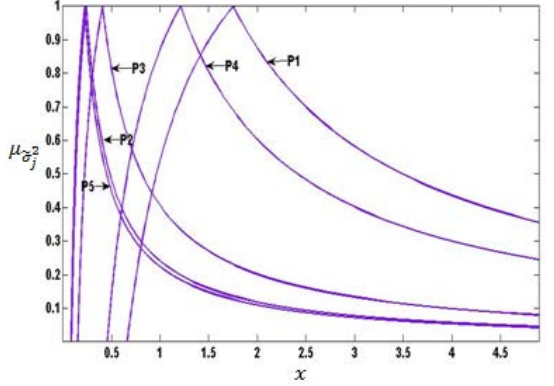

Fig. 4. Triangular fuzzy numbers of the variances of profiles 1 to 5

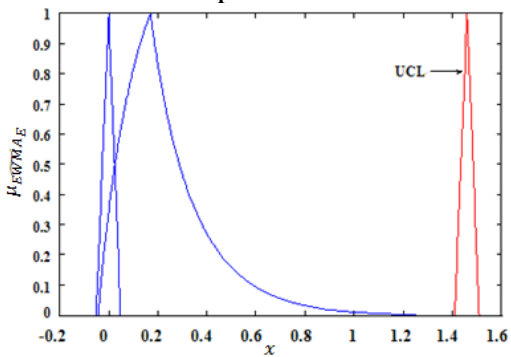

Fig. 7. FEWMA control chart for variance

From sample number 27 to the end, the hypothesis of being in control, is rejected. The almost long sequence of "no decision" result, is a warning signal about the necessity of more checking. So from sample 22 to the end, there is a warning of probable exit of slop control chart from in control status which requires more accurate check. It can be argued that data analysis based on the proposed fuzzy quality control charts lead to better and more accurate results, because of its ability of covering the uncertainty of parameter estimation. 


\section{Conclusion and Further Research}

Due to the fact that in many real-world problems, data are vague and uncertain, the assumption of crisp profiles for processes is not realistic. Fuzzy set theory is an efficient tool to address this shortcoming. In this paper, a new method of identifying small changes of process profile has been proposed in which profile parameters were assumed fuzzy and vague. For this purpose, we have developed Fuzzy EWMA control charts and discussed the efficiency of proposed method by solving an example. The results have shown that this method was highly efficient in discovering assignable causes in profiles. For further research, fuzzy profiles in more complex structures, e.g. polynomial, nonlinear, extended linear, etc., in phase I and phase II are to be addressed

\section{References}

Bucklly, J. J. (2006). Fuzzy probability and statistics. New York: Springer Berlin Heidelberg.

Croarkin, C., \& Varner, R. (1982). Measurement Assurance for Dimensional Measurements on Integrated-Circuit Photomasks. NBS Technical Note 1164.

Eyvazian, M., Noorossana, R., Saghaei, A., \& Amiri, A. (2011). Phase II monitoring of multivariate multiple linear regression profiles. Quality and Reliability Engineering International, 27(3), 281-296.

Fazel Zarandi, M., \& Alaeddini, A. (2010). Using Adaptive Nero-Fuzzy Systems to Monitor Linear Quality Profiles. Journal of Uncertain Systems, 4(2), 147-160.

Ghobadi, S., Noghondarian, K., Noorossana, R., \& Sadegh Mirhosseini, S. M. (2012). Developing a multivariate approach to monitor fuzzy quality profiles. Quality \& Quantity, 48(2), 817-836.

Hosseinifard, S., Abdollahian, M., \& Zeephongsekul, P. (2011). Application of artificial neural networks in linear profile monitoring. Expert Systems with Applications, 38(5), 4920-4928.

Kang, L., \& Albin, S. (2000). On-line monitoring when the process yields a linear profile. Journal of Quality Technology , 32(4), 418-426.

Kim, K., Mahmoud, M., \& Woodall, W. (2003). On the monitoring of linear profiles. Journal of Quality Technology, 35(3), 317-328.

Li, Z., \& Wang, Z. (2010). An exponentially weighted moving average scheme with variable sampling intervals for monitoring linear profiles. Computer \& Industrial Engineering, 59(4), 630-637.

Moghadam, G., Raissi Ardali, G., \& Amirzadeh, V. (2015). Developing new methods to monitor phase II fuzzy linear profiles. Iranian Journal of fuzzy systems . In press.

Montgomery, D. (2009). Introduction to Statistical Quality Control. New York: John Wiley and Sons.

Niaki, S., Abbasi, B., \& Arkat, J. (2007). A generalized linear statistical model approach to monitor profiles. International Journal of Engineering, Transactions A: Basics, 20(3), 233-242.

Noghondarian, K., \& Ghobadi, S. (2012). Developing a univariate approach to phase-I monitoring of fuzzy quality profiles. International Journal of Industrial Engineering Computations, 3(5), 829-842.

Noorossana, R., Amiri, A., Vaghefi, S., \& Roghanian, E. (2004). Monitoring quality characteristics using linear profile. $3^{\text {rd }}$ International Industrial Engineering Conference. Tehran.

Noorossana, R., Eyvazian, M., \& Vaghefi, S. A. (2010). Phase II monitoring of multivariate simple linear profiles. Computers and Industrial Engineering, 58(4), 563-570.

Noorossana, R., Saghaie, A., \& Amiri, A. (2011). Statistical Analysis of Profile Monitoring. (I. Hoboken, Ed.) New Jersey: John Wiley and Sons.

Saghaei, A., Mehrjoo, M., \& Amiri, A. (2009). A CUSUM-based method for monitoring simple linear profiles. The International Journal of Advanced Manufacturing Technology, 45(11), 1252-1260.

Zhang, J., Li, Z., \& Wang, Z. (2009). Control chart based on likelihood ratio for monitoring linear profiles. Computational Statistics and Data Analysis, 53(4), 1440-1448.

Zou, C., Zhang, Y., \& Wang, Z. (2006). Control chart based on change-point model formonitoring linear profiles. IIE Transactions, 38(12), 1093-1103.

Zou, C., Zhou, C., Wang, Z., \& Tsung, F. (2007). A self-starting control chart for linear profiles. Journal of Quality Technology, 39(4), 364-375. 